\title{
Assessment of drug-induced arrhythmic risk using limit cycle and autocorrelation analysis of human iPSC-cardiomyocyte contractility
}

\author{
R. Jason Kirby ${ }^{1 \#}$, Feng Qi2 ${ }^{2 \#}$, Sharangdhar Phatak ${ }^{1 \#}$, Layton H. Smith ${ }^{1}$, Siobhan Malany ${ }^{1 *}$ \\ Sanford Burham Prebys Medical Discovery Institute \\ ${ }^{1}$ Conrad Prebys Center for Chemical Genomics \\ ${ }^{2}$ Applied Bioinformatics Facility \\ 6400 Sanger Rd, Orlando, Florida, USA 32827 \\ \#Authors contributed equally to the work. \\ *Corresponding author: Siobhan Malany, Ph.D. \\ email: smalany@ sbpdiscovery.org \\ Tel.: 407-745-2000 Ext.2130
}

(C) 2016. This manuscript version is made available under the Elsevier user license 


\begin{abstract}
Cardiac safety assays incorporating label-free detection of human stem-cell derived cardiomyocyte contractility provide human relevance and medium throughput screening to assess compound-induced cardiotoxicity. In an effort to provide quantitative analysis of the large kinetic datasets resulting from these real-time studies, we applied bioinformatic approaches based on nonlinear dynamical system analysis, including limit cycle analysis and autocorrelation function, to systematically assess beat irregularity. The algorithms were integrated into a software program to seamlessly generate results for 96-well impedance-based data. Our approach was validated by analyzing dose- and time-dependent changes in beat patterns induced by known proarrhythmic compounds and screening a cardiotoxicity library to rank order compounds based on their proarrhythmic potential. We demonstrate a strong correlation for dose-dependent beat irregularity monitored by electrical impedance and quantified by autocorrelation analysis to traditional manual patch clamp potency values for hERG blockers. In addition, our platform identifies non-hERG blockers known to cause clinical arrhythmia. Our method provides a novel suite of medium-throughput quantitative tools for assessing compound effects on cardiac contractility and predicting compounds with potential proarrhythmia and may be applied to in vitro paradigms for pre-clinical cardiac safety evaluation.
\end{abstract}

Key words: human induced pluripotent stem cell cardiomyocytes (hiPSC-CMs); arrhythmia; label-free impedance; autocorrelation; limit cycle; Python v2.7 software 


\section{Introduction}

Novel higher throughput methods for quantifying drug-induced proarrhythmic cardiovascular risk are needed to better assess potential drug liabilities in relation to efficacy and increase the utility of in vitro models of human toxicity in early stage drug discovery and development. The implementation of human induced pluripotent stem cell-derived cardiomyocytes (hiPSC-CMs) into pre-clinical pharmacological studies has provided a relevant screening platform and a cost-effective alternative to animal models for preclinical screening of drug-induced cardiac risk. Monitoring of stem cell-derived cardiomyocytes using multi-electrode array (MEA) and electrode impedance real-time cell analysis (RTCA) has offered a powerful method to assess cardiac risk of newly developed pharmaceutical compounds. Both approaches monitor real-time compound-specific arrhythmic events associated with modulation of ion channels and provide comparable results across a panel of known proarrhythmic compounds (Guo et al., 2011; Xi et al., 2011; Abassi et al., 2012; Guo et al., 2013; Harris et al., 2013; Pradhapan et al., 2013; Wang et al., 2013; Nozaki et al., 2014; Gilchrist et al., 2015; Nozaki et al., 2016). These electrode-based platforms provide complementary datasets describing druginduced effects on cardiomyocyte function to methods such as manual and automated patch clamp techniques (Kramer et al., 2013; Gibson et al., 2014), calcium flux (Sirenko et al., 2013) and microphysical systems (MPS) (Mathur et al., 2015) and as we show herein, for RTCA, are predictive of drug-induced arrhythmogenic risk and can be implemented into early stage screening platforms to be confirmed by the lower through-put whole cell electrophysiology and tissue analysis.

Several groups have tested structurally and mechanistically diverse cardioactive compounds on hiPSC-CMs employing label-free technology and demonstrate the approach is 
sensitive to arrhythmogenic drugs (Anson et al., 2011; Jonsson et al., 2011; Kolaja, 2014), providing comparable electrophysiology and pharmacology to data reported for primary human cardiac myocytes (Peng et al., 2010). Although hiPSC-CMs exhibit a transcriptional phenotype more similar to fetal heart than adult (Synnergren et al., 2012), convergence of hiPSC-CM technology and microelectrode label-free recording systems provide an integrated in vitro testing platform for human cellular studies amenable to early stage screening that aims to emulate in vitro and in vivo electrophysiological studies (Scott et al., 2014).

The utility and application of human label-free cellular studies for pharmaceutical toxicological screening is hindered by non-standard and low-throughput software analysis procedures. Methods such as Poincaré analysis characterize the firing patterns and stability of cardiomyocytes based on interbeat intervals (IBIs) (Kamen et al., 1996; Bergfeldt and Haga, 2003). However, the lack of temporal information is a primary limitation of the standard descriptors used in Poincaré measurements. Alternative methods to Poincaré plots are phasespace reconstruction, which employs a time delay to reconstruct the time series (Takens, 1981), and detrended fluctuation analysis, that determines the statistics of a repeating, non-stationary process (Kantelhardt et al., 2001). These approaches have been proposed based on nonlinear and dynamic analysis to quantify the IBIs; however, neither method is adaptable for high-throughput processing.

To this end, we have uniquely applied limit cycle and autocorrelation function to visualize and quantitate oscillatory irregularities of impedance-based contractility patterns generated by hiPSC-CMs. Limit cycles have been used to describe the dynamics of cell cycle and circadian rhythm (Gonze et al., 2002; de la Fuente, 2010) and autocorrelation function has been used to describe spatiotemporal patterns for pendulum arrays and signal processing (Qi et 
al., 2003a; Bendor et al., 2012). The mathematical models provide distinct information as to pattern type and persistence over the time series. Application of these computational approaches to cardiomyocyte contraction provides a novel and powerful tool for assessing proarrhythmic risk.

We validated our methods by analyzing changes in beat patterns induced by astemizole, dofetilide, and cisapride, known proarrhythmic compounds that exhibit impedance-based irregular beating (Guo et al., 2013; Wang et al., 2013). Furthermore, we integrated our bioinformatic approaches into a stand-alone Windows-based program to perform highthroughput limit cycle and autocorrelation analysis of cardiomyocyte contractility patterns and have applied this approach to screening the Enzo cardiotoxicity library. By applying autocorrelation to drug-induced beat irregularity patterns, we identify the known hERG inhibitors present in the Enzo library that likewise exhibit clinical arrhythmic risk with good correlation, rank order and accuracy as compared with $\mathrm{IC}_{50}$ values from both manual and automated patch clamp studies. We further identified irregular beat patterns induced by sotolol and alfuzosin, two drugs that are not identified by the hERG assay but are known to cause clinical arrhythmia (Yap and Camm, 2003; Lacerda et al., 2008). Lastly, we present a case study for early risk assessment based on our approach of a G-protein receptor targeted compound during lead optimization.

Uncharacterized drug entities that inhibit hERG channels are often terminated preclinically. However, not all compounds that inhibit the hERG channel result in QT prolongation or induce torsades de pointes (TdP) and some drugs that do not affect hERG can prolong QT and lead to TdP (Lu et al., 2008). Therefore, additional screening paradigms are needed to evaluate proarrhythmic compounds. Our automated drug cardiotoxicity computational assessment 
platform using mathematical models bridges hiPSC-CM technology and microelectrode recording systems with high throughput processing and quantitative analysis. Our results demonstrate sensitivity to hERG and non-hERG blockers and are correlative to data obtained from patch clamp studies. Thus, our software program applied to microelectrode recordings of iPSC-CMs can be seamlessly implemented into pre-clinical pharmacological workflows to evaluate proarrhythmic compounds earlier in drug development to be validated by in vitro and in vivo electrophysiology and MPS based studies .

\section{Materials and Methods}

\section{Reference compounds}

Dofetilide (Sigma-Aldrich, St. Louis, MO), astemizole, and cisapride (Enzo Life Sciences, Farmingdale, NY) were prepared in $100 \%$ dimethylsulfoxide at $10 \mathrm{mM}$ and stored in 96 -well polypropylene plates at $-80^{\circ} \mathrm{C}$. ScreenWell Cardiotoxicity Library (Enzo Life Sciences, Farmingdale, NY) containing 130 compounds were plated at $10 \mathrm{mM}$ in DMSO and stored at $80^{\circ} \mathrm{C}$.

\section{Cardiomyocyte cell culture}

Cryopreserved hiPSC-CMs (Cellular Dynamics International, Madison, WI, CDI \#CMC100-010-001) were thawed in plating media supplied by the vendor and approximately $3 \times 10^{4}$ cells/well plated directly onto E-Plate Cardio (ACEA Biosciences, San Diego, CA) precoated with $0.01 \mathrm{mg} / \mathrm{mL}$ fibronectin in PBS for $3 \mathrm{~h}$ at $37^{\circ} \mathrm{C}$. Cells were cultured for 14 days at $37^{\circ} \mathrm{C}, 5 \%$

$\mathrm{CO}_{2}$ to ensure synchronous batches of cells. The maintenance media (CDI) was changed every 2 days after plating and $24 \mathrm{~h}$ prior to dosing using a Viaflo 96 channel pipettor (INTEGRA Biosciences Hudson, NH) placed in the tissue culture hood. 


\section{Impedance measurements}

Viability and contractility of cardiomyocytes were monitored by impedance using the xCELLingence RTCA (real-time cell analyzer) Cardio system (ACEA Biosciences, San Diego, CA). Impedance was measured for 5 consecutive 60-s sweeps (recorded at a sampling rate of $12.9 \mathrm{~ms})$ at selected time points and reported as cell index $(\mathrm{CI})$. Prior to drug treatment, a baseline was recorded to ensure the cells established a beat rate of 40-60 beats/min. Drug stocks were prepared at $10 \mathrm{mM}$ stock concentration and serial diluted in $100 \%$ dimethylsulfoxide and further diluted in maintenance media in a separate $96-$ well plate at $7 \mathrm{x}$ target concentration. The drug plate was equilibrated to $37^{\circ} \mathrm{C}$ prior to diluting $1: 7$ into the E-plate using the Viaflo 96 channel pipet to give a final medium volume of $175 \mu \mathrm{l} /$ well in $0.1 \%$ DMSO. All compounds were tested in duplicate on two separate E-plates seeded with cells from separate thaws.

\section{Data acquisition, handling and statistical analysis}

Cell index values were normalized both to the time point just prior to compound treatment and to the mean cell index of DMSO control wells. Normalized cell index values for all 96 wells were exported in list format as tab-delimited text files for each of five consecutive 60 s sweeps prior to compound addition and at $1 \mathrm{~h}, 4 \mathrm{~h}$, and $24 \mathrm{~h}$ following treatment. The 5 exported RAW files associated with each time point are suffixed with "_(1-5)min” to identify the 5 sweeps, and placed in a directory for each time point. Statistical analysis and graph generation was performed with GraphPad Prism version 7.0 for Windows (GraphPad, La Jolla, CA, USA). Dose-response experiments were analyzed by non-linear regression (four parameters). Experimental data were 
statistically analyzed as indicated for each experiment. The data reported are individual values or rounded means and corresponding standard deviation (SD).

\section{Data Analysis}

Normalized cell index values are smoothed using moving windows of size 10 time interval of output series (default time interval of instrument is 12.9ms). Limit Cycle Analysis - 2dimensional phase portrait of the IBIs were plotted as the amplitude vs. the first derivate for each recorder. Autocorrelation Analysis - Autocorrelation is calculated as the cross-correlation of the cell index signal with itself with different time lag, which was implemented using the autocorrelation function for one-dimensional arrays (time series) from the StatsModels library. For every 1-min sweep and every well, characteristic correlation time, tau is calculated in two steps as follows: first, the sum of the squared values of auto-correlation for that well is calculated, then result is normalized by the length of the data series for the given well (4633 data points).

\section{Software development and automation}

A stand-alone Windows (only) based tool to perform high-throughput limit-cycle and tau analysis was primarily developed using Python v2.7. Other open-source libraries, e.g., PySide (www.pyside.org), Numerical Python v1.9.2 (www.numpy.org), and matplotlib v1.4.3 (www.matplotlib.org), were used to develop a simple graphical user interface, perform mathematical calculations and generate plots, respectively. The tool was designed to process RAW data of up to four time points, each time point consisting of fixed 5-min sweeps for a 96well plate format from the ACEA xCELLigence RTCA Cardio system. The 5 RAW files

associated with 1 time point are suffixed with “_(1-5)min”, to identify the 5 sweeps, and placed 
in a directory. The GUI allows the end-user to choose the directory, along with simple dropdown options for output image resolution, image type, an option to scale limit-cycles based on a threshold cutoff value obtained from the adjacent slider and 4 options for smoothing the initial RAW data. The output will consist of images of limit cycles per individual well and a complete 96 well-plate view, tau values per plate as flat text (.csv) files, and heat map plots of the average tau values for the entire plate and a composite image consisting of the image of limit-cycles and heat map of tau values in a plate-view format. All the output files will be saved in the directory of the chosen time point. An executable of the program along with sample data and results is available upon request and it has no additional dependencies. The software was developed and tested on a machine with Windows 7 Pro (SP1), 16 G.B. RAM. The software program is available for download as an executable file complete with protocol and demonstration.

\section{Results}

Assessing Beat Rate Dynamics with Limit Cycle and Autocorrelation Analysis

Human iPSC-CMs obtained commercially as cryopreserved products are highly pure cell populations that exhibit normal cardiomyocyte physiology and expression of specific cardiac biomarkers. The cardiomyocytes spontaneously beat several days after plating, and are viable in culture for an extended time making them amenable to medium-throughput testing (Hartman et al., 2015). Cardiomyocyte beating was monitored on the RTCA Cardio system which records impedance transients referred to as cell index (CI) values correlated to functional beating activity; average CI values provide an indication of cell viability (Guo et al., 2011). Human iPSC-CMs maintained for 14 days in culture exhibited synchronous beating and stable, saturating CI values recorded over $24 \mathrm{~h}$. 
To evaluate the influence of known proarrhythmic drugs on the contractile activity of hiPSC-CMs, we characterized dose-dependent and time-dependent effects of dofetilide, astemizole, and cisapride on cardiomyocyte beat pattern and regularity. These drugs belong to distinct drug classes based on their primary activity but are hERG channel blockers with known cardiac liabilities (Redfern et al., 2003). Human iPSC-CMs incubated with $0.12 \mu \mathrm{M}$ of each of the compounds for one hour exhibit drug-induced irregular impedance traces reminiscent of arrhythmias associated with TdP compared to control cells (Fig. 1a). No significant effects (< $5 \%$ decrease) on the average CI were observed indicating the beat pattern disruptions are due to cardiomyocyte ion-channel specific events and not due to general cytotoxicities. Dose-dependent changes in beat rhythm cannot be accurately quantified without taking into account the effects of the irregular beat patterns. Current methods to determine cardiac risk rely on subjective identification of peaks that can be ambiguous in cases of arrhythmia where numerous minimal index fluctuations are observed. We focused on these drugs as case studies to validate our bioinformatic approach to assess beat rate dynamics using limit cycle and autocorrelation function.

Limit cycle phase plots were generated from impedance data reflecting cardiomyocyte contractile state every $\mathbf{1 2 . 8 m s}$ and recorded over 300 s to identify variation in the number of periodic oscillations following drug treatment of hiPSC-CMs. The phase plots were graphed by plotting the change in velocity, i.e. the first derivative $(d y / d t)$, against the instantaneous cell index position (y). The first derivative was calculated using the finite difference approximation: $V_{t}=\frac{y(t+\Delta t)-y(t)}{\Delta t}$, here the $\Delta t$ is the sample time interval $(12.9 \mathrm{~ms})$. Control treatment yields a stable oscillation that traces the closed phase trajectory and approaches the limit cycle as $t$ approaches infinity (Fig. 1b). Treatment with the proarrhythmic compounds caused a deviation 
from the phase trajectory, creating new dynamic patterns which are illustrated by additional limit cycles with varying amplitude and duration (Fig. 1b).

Although the limit cycle approach is intuitive to present the most important feature of the dynamical systems, we utilize the approach here for the purposes of visual comparison. In order to quantify the decay of beat rate regularity, we implemented the autocorrelation function, derived from nonlinear dynamics, to model the contraction time series (Qi et al., 2003b; Qi et al., 2003a). The characteristic correlation time (Pikovsky et al., 1997), measures the regularity of the time series of a dynamical system and is based on the normalized autocorrelation function $c(\tau)$, defined by $c(t)=\frac{<\tilde{x}(t) \tilde{x}(t+\tau)>}{<\tilde{x}^{2}(t)>}$. Here $x(t)$ is the instantaneous position at time $t, \tau$ is the time delay, and $\tilde{x}(t)=x(t)-<x(t)>$. The autocorrelation functions were generated by calculating the cross-correlation of the cell index signal with itself at different sampling times. The characteristic correlation time is then evaluated as $\tau_{c}=\frac{1}{T} \int_{T} c^{2}(\tau) d \tau$. The resulting plot of the correlation coefficient $\left(\tau_{c}\right)$ versus time $(t)$ depicts the similarity between observations as a function of the time lag between them which provides greater temporal information than the Poincaré plot. Control treatment yields a strong autocorrelation that decays slowly with time and results in a highly ordered periodic correlation; whereas, treatment with the proarrhythmic drugs yields a weak autocorrelation that decays rapidly with significant noise (Fig. 1c). The average of the autocorrelation function applied to five sequential 60 s impedance recordings results in a calculated order parameter (tau).

These results highlight the following: (1) limit cycle models changing oscillations of the hiPSC-CMs exposed to drug and identifies the number of oscillatory cycles indicative of the complexity of the arrhythmic pattern and (2) the tau value from autocorrelation function quantifies the persistence of repeating oscillations and a decrease in value induced by 
proarrhythmic compounds compared to control indicates a quantifiable decrease in regularity of the beat pattern.

\section{Dose-Dependent Changes in Tau Provide Quantitative Assessment of Beat Irregularity}

Our bioinformatic approaches provide a quantitative system that can be used to rank order drug candidates based on their impedance-detected arrhythmias. To demonstrate this, we determined average tau values for hiPSC-CMs treated for $1 \mathrm{~h}$ with a range of drug concentrations and normalized results to values obtained for control-treated cells. Results were fit to non-linear regression curves to extract a tau $\mathrm{EC}_{50}$ (the drug concentration that decreased tau by $50 \%$ ) for beating irregularity. By applying autocorrelation to the concentration-response data, dofetilide, astemizole, and cisapride exhibited tau $\mathrm{EC}_{50}\left(\mathrm{pEC}_{50} \pm \mathrm{SD}\right)$ values of $1.5 \mathrm{nM}(8.81 \pm 0.10), 7.2 \mathrm{nM}$ $(8.14 \pm 0.12)$ and $14.1 \mathrm{nM}(7.70 \pm 0.14)$, respectively (Fig. 2). The data is averaged from three separate cell thaws showing the robustness and reproducibility of the tau determination. The rank order of compounds is equivalent to comparing the lowest concentration of compound that resulted in greater than $20 \%$ irregular beats over $1 \mathrm{~min}\left(\mathrm{IB}_{20}\right.$ values) reported in the literature to be 3nM, 30nM, and 300nM, respectively (Guo et al., 2011). Similar to $\mathrm{IB}_{20}$ values, tau $\mathrm{EC}_{50}$ values can be used to provide additional quantitative information across the concentration range based on an automated calculation. Importantly, tau $\mathrm{EC}_{50}$ values are independent of a set threshold for peak identification and can be compared at various time points post treatment.

Prior to applying the algorithms for limit cycle analysis and autocorrelation, we subjected the raw $\mathrm{CI}$ values to an unweighted moving average to smooth fluctuations in the time series. We arbitrarily choose 10 as the size of the smoothing window. To ensure that the factor reduced noise but does not significantly affect outcome, we compared results for limit cycle and 
autocorrelation applying smoothing windows for 1, 5, 10, and 20 and present the data for cisapride in the supplemental material (Supplementary Fig. 1). Resulting tau values for compound doses were inversely proportional to the smoothing window size, yet concentrationresponse curves were equivalent when tau values were normalized to untreated controls. Nonlinear regression analysis for each curve resulted in a tau 50 value for cisapride of $14 \mathrm{nM}$ regardless of the smoothing factor applied indicating that our conditions for determining tau are not dependent on the smoothing factor and applying a factor of 10 provides a limit cycle plot with less visual distortion.

\section{High-Throughput Analysis of Impedance-Detected Arrhythmias}

In an effort to provide a robust and seamless procedure for analyzing real time impedancedetected arrhythmias for screening data, we designed a stand-alone Windows-based software solution to directly process unaltered output files from the RTCA Cardio system for up to four user-defined time points. The executable tool processes raw data to generate the following: (1) images of limit cycles per individual well, (2) tau values per plate as text files, and (3) a composite image consisting of the image of limit cycles and a heat map of tau values in a 96-well plate-view format.

We screened the Enzo library of 130 cardioactive and nontoxic controls in the contractility assay and implemented our software program to highlight the validity and functionality of our method. The raw data files containing the changes in CI for hiPSC-CMs over $24 \mathrm{~h}$ were imported into the software program and results were generated for pre-treatment and 1 , 4, and $24 \mathrm{~h}$ post-treatment data. The hiPSC-CM impedance traces, limit phase plots, and tau heat maps for pre- and $4 \mathrm{~h}$ post-treatment with compounds tested at $10 \mu \mathrm{M}$ are shown in Figure 3 . 
The limit cycle phase plots provide a visual representation for well-to-well consistency in beating rhythm in the pre-treated iPSC-CMs (Fig. 3c) and flags wells that exhibit distinct oscillations after compound exposure (Fig. 3d). Proarrhythmic compounds exhibit limit cycle plots with additional oscillation patterns; whereas, the limit cycle flattens in cases where beating ceases. The tau values provide a quantitative value for beat rhythm persistence. Tau values determined prior to compound treatment (Fig. 3e) were uniform across a single plate with a mean tau value of $0.046 \pm 0.009(\mathrm{~N}=96$ wells). These values are consistent plate-to-plate providing an average tau of $0.047 \pm 0.003$ ( $\mathrm{N}=9$ plates) and serve as quality control prior to treatment. Wells that exhibit a decrease in tau value, post-treatment, identify compounds that induce arrhythmia as shown in blue in Figure 3f. Tau values are sensitive to minor fluctuations in beat consistency, therefore, comparison of pre- and post-treatment for both the phase plots and tau values and replicate analysis will help in discriminating false positives from persistent cardiac arrhythmias.

A screen using the RTCA cardio assay tested at $10 \mu \mathrm{M}$ compound concentration identified 69 compounds to have effects on cardiomyocyte viability, contraction amplitude, or beat rate. Results for 131 compounds tested at 1h, 4h, and 24h are listed in Supplemental Table 1. Characteristic impedance traces for proarrhythmic drugs, known to induce TdP and prolonged QT interval, and their corresponding unique limit cycle phase plots are shown in Supplementary Fig. 2. Risperidone is included as an example of a proarrhythmic compound identified in the library that showed a concentration-dependent and time-dependent decrease in beat rate and amplitude. Impedance traces, limit cycle analyses and a nonlinear curve fit of the tau values over the concentration range is also provided in supplemental material (Supplementary Fig. 3a-c). 
These results provide a more reliable description of the data than plots for beat rate and amplitude.

From the results of our screen, 10 compounds were flagged as proarrhythmic based on their effect on the limit cycle and tau values. An additional 11 compounds that stopped cardiomyocyte beating at $10 \mu \mathrm{M}$ were found to induce arrhythmia when tested in concentrationresponse. These compounds are listed in Table 1 along with their classification, tau $\mathrm{IC}_{50}$ values determined from autocorrelation function, $\mathrm{IC}_{50}$ values reported in the literature from manual and automated patch clamp studies, and whether the drugs are reported to have $(+)$ or not have (-) occurrence of QT prolongation or TdP in the clinic. We also tested known proarrhythmics E4031, ibutilide and alfuzosin and include results in Table 1. A correlation graph of the $\mathrm{pEC}_{50}$ values determined by autocorrelation versus those determined by patch clamp for hERG inhibition is shown in Figure 4A. A Pearson correlation analysis resulted in statistically significant correlation $(\mathrm{r}=0.7882$ and $0.8345, \mathrm{p}<0.0001$ for manual and automated patch clamp reported values, respectively); the comparison indicates our assay platform is selective and sensitive to hERG inhibitors.

Drugs absent from the correlation but listed in Table 1 include: (1) verapamil which showed a dose-dependent increase in tau corresponding to increased beat rate; the drug does not exhibit clinical liabilities; (2) mexiletine and sibutramine, which had no effect on tau; these drugs exhibit weak hERG inhibition and no reported TdP liabilities; (3) chlorpromazine, which stops beating below the reported $\mathrm{IC}_{50}$ value for hERG inhibition; (4) amiodarone, bepridil, and imipramine, which also had no effect on tau; these drugs inhibit hERG and have reported cardiac liabilities and are false negatives in our assay; and (5) sotalol and alfuzosin which induce irregular beating in the impedance assay and dose-dependently decrease tau. These drugs 
increase the risk of QT elongation and clinical arrhythmia but are not identified by hERG patch clamp assays. Impedance traces illustrating beat irregularity induced by alfuzosin are shown in Figure $4 \mathrm{~b}$ and concentration-response curves show alfuzosin decreased tau with $\mathrm{EC}_{50}=3.9$ and $6.8 \mu \mathrm{M}$ at $1 \mathrm{~h}$ and $4 \mathrm{~h}$, respectively (Figure $4 \mathrm{c}$ ). Taken together, our assay system is sensitive to both hERG and non-hERG arrhythmogenic compounds with an $86 \%$ accuracy rate.

Bioinformatic Method for Early Risk Assessment during Lead Optimization: Case Study

We next tested the application of our method to assess chemical candidates for potential cardiac liabilities during lead optimization. As a case study, we show results for a $G$ protein-coupled receptor agonist with distinct mode of action from drugs listed in Table 1. The proprietary compound was identified after structure-activity relationship studies as a candidate for an in house heart failure disease program. Exposure of hiPSC-CMs to the compound for $4 \mathrm{~h}$ at $10 \mu \mathrm{M}$ caused a small but stable irregularity in the beating rhythm (Fig. 5a). A break in the limit cycle phase plot was observed indicating the existence of two distinct oscillations (Fig. 5b). The autocorrelation plot showed two persistent patterns that decayed over time resulting in a tau value of 0.018 and corresponding $63 \%$ decrease at $10 \mu \mathrm{M}$ as compared to an average tau value of $0.048 \pm 0.006$ for control treatment $($ Fig. 5c). The compound was subsequently tested in the automated Q-patch-based hERG assay and determined to have an $\mathrm{IC}_{50}$ value of $2.2 \mu \mathrm{M}$ (Fig. 5d). The $\mathrm{IC}_{50}$ value was 1000 -fold higher than the $\mathrm{IC}_{50}$ value determined in an in vitro cAMP signaling assay. Nonetheless, the chemical scaffold was flagged early in lead optimization having both beat irregularity and hERG inhibition, and was subsequently eliminated from further consideration as a clinical candidate. 


\section{Discussion}

We present herein, our validated software solutions for automated high-throughput in vitro drug cardiotoxicity assessment based on limit cycle analysis and autocorrelation function. We validated our approach as a tool for assessing proarrhythmic risk of cardioactive compounds and demonstrated the versatility and throughput of our method as a screening platform to provide rapid and quantitative analysis of large kinetic datasets collected from real-time cell recordings. The techniques together offer qualitative information of the dynamic time series for contracting cardiomyocytes and we provide a quantitative assessment indicating the specificity, sensitivity, and predictability of our novel approach relative to current methods to screen, detect, and rank drugs for adverse cardiac arrhythmias.

Limit cycle analysis of the impedance-detected arrhythmias provides full dynamic information as to discrete oscillations with particular amplitude induced by proarrhythmic drugs. Limit cycle plots show changes in the phase space which can be used to identify aberrant oscillatory patterns embedded in the time series. Plotting the first derivative of the time series allows limit cycles to be standardized to the same scale and data can be qualitatively compared across different platforms and instruments.

Our application of autocorrelation function to the cardiomyocyte contraction series results in a single quantitative value to characterize the persistence of the repeating pattern. The autocorrelation function provides a robust alternative to analyzing the time series by Poincaré plot that captures partial nonlinear aspects of the data sequence. The standard descriptors used in quantifying the Poincaré plot (SD1, SD2) are limited to measurement of the dispersion in two directions. Complex correlation measure represents an extension of the Poincaré plot to higher dimensions by incorporating lag times of the IBIs; however the information captured from the 
analysis is difficult to compare across samples (Karmakar et al., 2009). Autocorrelation compares the entire sequence at different time points and thus collectively incorporates the time lags based on the IBIs.

Together the bioinformatic approaches applied to real-time cellular recordings offers high throughput processing, ease of analysis, and enhanced quantitative modeling of in vitro induced cardiac arrhythmia. Each provides distinct but complementary information about the arrhythmia with limit cycle characterizing the complexity of the beat pattern and autocorrelation quantifying beat regularity. To increase the functionality and accessibility of our method for pharmacologists, we have integrated the approaches into a software program that processes $\sim 8.8$ million raw data points extracted at four time points post-treatment in a few minutes and deposits per well data in a user defined directory. The automated processing increases the throughput for assessing in vitro beat irregularity. We evaluated 131 drugs in the Enzo library and identified 26 drugs that induce beat irregularity after $4 \mathrm{~h}$ exposure to iPSC-CMs. Determination of tau $\mathrm{EC}_{50}$ values for this set of drugs provides proof of concept that our approach is predictive for detecting drugs associated with clinical arrhythmias with $86 \%$ accuracy.

The novelty of this study is the application of limit cycle and autocorrelation analyses to RTCA data providing novel, semi-automated method to observe deviation in contraction rhythmicity that as we show in Table $\mathbf{1}$ and Fig. $\mathbf{4}$ is correlative to traditional patch clamp data and predictive of hERG and non hERG inhibiting drugs that potentially cause QT prolongation. This method combined with data sharing and visualization programs provides a powerful platform that can be integrated into a broader paradigms such as the Comprehensive Proarrhythmia Assay initiative that seeks to lower drug attrition rates and development costs by providing a more comprehensive assessment of safety liabilities earlier in the drug development 
process that corroborate human in vitro and in vivo electrophysiological data (Sager et al., 2014).

The program is available as an executable file and can be used as a standalone program to analyze beat patterns and potentially be modified to analyze real-time calcium flux responses or action potentials. Our automated high-throughput approach is a powerful qualitative and quantitative method to rank order compounds according to their predictive cardiac risk and aims to bridge the gap between assay throughput and clinical relevance.

\section{Acknowledgements}

This work was funded by the Department of the Air Force (FA8650-13-2-6453 to Smith, L.) and the Florida Translational Research Program (Florida Department of Health contract COHG6 to Smith, L). The views expressed are those of the authors and do not necessarily reflect the official policy or position of the Air Force, the Department of Defense, or the U.S. Government.

\section{Author Contributions}

R.J.K. designed and performed experiments, analyzed data, prepared the manuscript, and helped to envision the final bioinformatics protocol. F.Q. envisioned and programmed the mathematical equations for limit cycle and autocorrelation and applied the program to data generated by R.J.K. S.P. integrated the mathematical equations developed by F.Q. and designed the Windows software program executable file complete with graphical user interface. L.H.S. is Principal Investigator of the Department of the Air Force and Florida Translational Research Program grants. S.M. is key investigator of the Department of the Air Force grant and Florida Translational Research Program, envisioned the automated, quantitative concept for analyzing contractility, supervised the work, and wrote the manuscript.

\section{Competing Financial Interests}

The authors declare no competing financial interests. 
Table 1.

Summary of tau $\mathrm{EC}_{50}$ values determined in iPSC-CMs

\begin{tabular}{|c|c|c|c|c|c|c|}
\hline Drug & Mode of Action & $\begin{array}{l}\text { Tau } \mathrm{EC}_{50} \\
(\mathrm{nM})\end{array}$ & $\begin{array}{l}\mathrm{I}_{\text {HERG }} \mathrm{IC}_{50} \\
(\mathrm{nM})^{\mathrm{a}}\end{array}$ & $\begin{array}{l}\text { Qpatch-HT/ } \\
\text { Patch Express } \\
\mathrm{IC}_{50}(\mathrm{nM})^{\mathrm{b}}\end{array}$ & $\mathrm{QT}^{\mathrm{c}}$ & $\mathrm{TdP}^{\mathrm{c}}$ \\
\hline Quinidine & \multirow{5}{*}{$\begin{array}{l}\text { Class I } \\
\text { antiarrhythmic }\end{array}$} & 800 & 410 & 720 & $(+)$ & $(+)$ \\
\hline E4031 & & 9.0 & 7.7 & 14 & $(+)$ & $(+)$ \\
\hline Disopyramide & & 2,000 & 7,300 & 14,400 & $(+)$ & $(+)$ \\
\hline Mexiletine & & $\mathrm{NE}$ & 3700 & NR & $(-)$ & $(-)$ \\
\hline Flecainide & & 1,340 & 3,900 & 1,500 & $(+)$ & $(+)$ \\
\hline Dofetilide & \multirow{4}{*}{$\begin{array}{l}\text { Class III } \\
\text { antiarrhythmic }\end{array}$} & 1.5 & 5 & 30 & $(+)$ & $(+)$ \\
\hline Ibutilide & & 1.4 & 12 & 18 & $(+)$ & $(+)$ \\
\hline amiodarone & & $\mathrm{NE}$ & 47 & 860 & $(+)$ & $(+)$ \\
\hline Sotalol & & 10,700 & $>100,000$ & 111,000 & $(+)$ & $(+)$ \\
\hline Cisapride & Prokinetic & 14 & 21 & 20 & $(+)$ & $(+)$ \\
\hline \multirow{2}{*}{$\begin{array}{l}\text { verapamil } \\
\text { bepridil }\end{array}$} & \multirow{2}{*}{$\mathrm{Ca}^{2+}$ blocker } & Increase & 143 & 250 & $(-)$ & $(-)$ \\
\hline & & $\mathrm{NE}$ & 290 & 160 & $(+)$ & $(+)$ \\
\hline alfuzosin & alpha 1 blocker & 6,800 & $\mathrm{NE}$ & NR & $(+)$ & $(+)$ \\
\hline Risperidone & \multirow{7}{*}{ Antipsychotic } & 140 & 167 & 260 & $(+)$ & $(+)$ \\
\hline Paliperidone & & 470 & 530 & 780 & $(+)$ & $(+)$ \\
\hline Thioridazine & & 1,360 & 33 & 500 & $(+)$ & $(+)$ \\
\hline Droperidol & & 311 & 77 & 60 & $(+)$ & $(+)$ \\
\hline Haloperidol & & 188 & 27 & 40 & $(+)$ & $(+)$ \\
\hline chlorpromazine & & $\mathrm{NE}$ & 21,000 & 1,500 & $(+)$ & $(+)$ \\
\hline Pimozide & & 14.3 & 18 & 40 & $(+)$ & $(+)$ \\
\hline \multirow{2}{*}{$\begin{array}{l}\text { Halofantrine } \\
\text { Ketoconazole }\end{array}$} & \multirow{2}{*}{$\begin{array}{l}\text { Antimalarial } \\
\text { /antifungal }\end{array}$} & 150 & 200 & 380 & $(+)$ & $(+)$ \\
\hline & & 3,750 & 1,700 & NR & $(+)$ & $(-)$ \\
\hline Orphenadrine & Anticholinergic & 430 & 850 & NR & $(-)$ & $(-)$ \\
\hline \multirow{2}{*}{$\begin{array}{l}\text { Sibutramine } \\
\text { Imipramine }\end{array}$} & \multirow{2}{*}{ SNRI } & $\mathrm{NE}$ & 3,900 & NR & $(+)$ & $(-)$ \\
\hline & & $\mathrm{NE}$ & 3,400 & NR & $(+)$ & $(+)$ \\
\hline Astemizole & \multirow{3}{*}{ Anti-histamine } & 7.2 & 1 & 4 & $(+)$ & $(+)$ \\
\hline Terfenadine & & 230 & 7 & 50 & $(+)$ & $(+)$ \\
\hline Hydroxyzine & & 438 & 180 & NR & $(-)$ & $(-)$ \\
\hline Nilotinib & \multirow{2}{*}{$\begin{array}{l}\text { Tyrosine kinase } \\
\text { inhibitor }\end{array}$} & 507 & 120 & 1,000 & $(+)$ & $(+)$ \\
\hline Sunitinib & & 1140 & 270 & 1,200 & $(+)$ & $(-)$ \\
\hline
\end{tabular}

NE: No effect; NR: Not reported 
a Patch clamp studies of human embroyonic kidney or Chinese hamster ovary cells overexpressing hERG channel (Aarons et al., 1998; Zhou et al., 1998; Teschemacher et al., 1999; Tie et al., 2000; Paul et al., 2002; Kirsch et al., 2004; Guo et al., 2005; Tarantino et al., 2005; Scholz et al., 2007; Luo et al., 2008; Huang et al., 2010; Zhang et al., 2010; Gintant, 2011; Lee et al., 2011; Vigneault et al., 2011; El Harchi et al., 2012; Crumb, 2014; Gualdani et al., 2015; Yun et al., 2015).

${ }^{\mathrm{b}}$ Automated patch clamp studies of human embroyonic kidney or Chinese hamster ovary cells overexpressing hERG channel (Kramer et al., 2013).

${ }^{\mathrm{c}}(+)$ and (-) represent positive or negative observations in the clinic for QT prolongation and TdP/arrhythmia (Redfern et al., 2003; Guo et al., 2013).

\section{Figure Legends}

Fig. 1. Limit cycle and autocorrelation analyses of impedance data monitoring cardiomyocyte contraction patterns following $1 \mathrm{~h}$ treatment with $0.1 \%$ DMSO or $0.12 \mu \mathrm{M}$ astemizole, dofetilide, or cisapride. (a) Representative impedance traces of normalized cell index values for 10s duration exhibit consistent beat rhythm in presence of DMSO and demonstrate an arrhythmic pattern induced by compound. (b) Limit cycle plots of velocity against the instantaneous position for normalized cell index values from five consecutive 60s sweeps. (c) Plots of autocorrelation function against the time delay for normalized cell index values from 60s sweep showing decay of beat regularity and the corresponding mean tau value $(\tau)$ for five consecutive 60 s sweeps.

Fig. 2. Concentration-dependent response of tau $(\tau)$ values calculated for cardiomyocyte beat patterns following $1 \mathrm{~h}$ treatment with (a) dofetilide, (b) astemizole, or (c) cisapride. Data are 
normalized to the mean of the DMSO controls and fit by nonlinear regression analysis to calculate tau $\mathrm{EC}_{50}$ values.

Fig. 3. Automated limit cycle and autocorrelation analysis of 96-well impedance data from the xCELLigence RTCA Cardio system for hiPSC-derived cardiomyocytes prior to drug treatment $(\mathrm{a}, \mathrm{c}, \mathrm{e})$ and $1 \mathrm{~h}$ post treatment with $10 \mu \mathrm{M}$ compounds form the Enzo cardiotoxicity library $(\mathrm{b}, \mathrm{d}, \mathrm{f})$. (a,b) Representative impedance traces of normalized cell index pre- and post-drug treatment, respectively. (c,d) Limit cycle phase plots of normalized cell index values from five consecutive 60s sweeps pre- and post-drug treatment, respectively. In wells where beating ceases, the phase plots collapses to straight line. $(\mathrm{e}, \mathrm{f})$ Heat map of tau values with gradient coloring from white $=$ 0.05 to dark blue $=0.005$ pre- and post-drug treatment, respectively. In wells where beating ceases, tau values are noted as not determined (ND).

Fig. 4. (a) Comparison of pEC50 values from autocorrelation analysis vs. hERG channel inhibition by manual patch clamp (closed circles) or automated Q-patch (open circles). (b) Impedance traces following treatment with decreasing concentrations of alfuzosin for $1 \mathrm{~h}$ and $4 \mathrm{~h}$. (c) Concentration response of autocorrelation (tau) values normalized to DMSO controls following alfuzosin treatment for $1 \mathrm{~h}$ (open circles) and $4 \mathrm{~h}$ (closed circles).

Figure 5. Effect of unknown test compound on cardiomyocyte beat regularity represented by (a) 10s impedance trace, (b) limit cycle plot and (c) plot of correlation coefficient vs. time and mean tau value from autocorrelation analysis of five consecutive 60s sweeps. (d) Compound inhibition of hERG activity was characterized by Q-patch-based hERG assay and performed at ChanTest. Data is mean of quadruplicate determinations. 


\section{References}

Aarons, L., Rowland, M., Khan, A., Taborelli, G., Ferrea, G., Tarantino, V., Fioredda, F., Rosina-Parenti, R., Cavenaghi, L., Borgonovi, M., 1998. Plasma and tonsillar tissue pharmacokinetics of teicoplanin following intramuscular administration to children. Eur J Pharm Sci 6, 265-270.

Abassi, Y.A., Xi, B., Li, N., Ouyang, W., Seiler, A., Watzele, M., Kettenhofen, R., Bohlen, H., Ehlich, A., Kolossov, E., Wang, X., Xu, X., 2012. Dynamic monitoring of beating periodicity of stem cellderived cardiomyocytes as a predictive tool for preclinical safety assessment. Br J Pharmacol 165, 1424-1441.

Anson, B.D., Kolaja, K.L., Kamp, T.J., 2011. Opportunities for use of human iPS cells in predictive toxicology. Clin Pharmacol Ther 89, 754-758.

Bendor, D., Osmanski, M.S., Wang, X., 2012. Dual-pitch processing mechanisms in primate auditory cortex. J Neurosci 32, 16149-16161.

Bergfeldt, L., Haga, Y., 2003. Power spectral and Poincare plot characteristics in sinus node dysfunction. J Appl Physiol (1985) 94, 2217-2224.

Crumb, W.J., Jr., 2014. Allosteric effects of erythromycin pretreatment on thioridazine block of hERG potassium channels. Br J Pharmacol 171, 1668-1675.

de la Fuente, I.M., 2010. Quantitative analysis of cellular metabolic dissipative, self-organized structures. Int J Mol Sci 11, 3540-3599.

El Harchi, A., Zhang, Y.H., Hussein, L., Dempsey, C.E., Hancox, J.C., 2012. Molecular determinants of hERG potassium channel inhibition by disopyramide. J Mol Cell Cardiol 52, 185-195.

Gibson, J.K., Yue, Y., Bronson, J., Palmer, C., Numann, R., 2014. Human stem cell-derived cardiomyocytes detect drug-mediated changes in action potentials and ion currents. J Pharmacol Toxicol Methods 70, 255-267.

Gilchrist, K.H., Lewis, G.F., Gay, E.A., Sellgren, K.L., Grego, S., 2015. High-throughput cardiac safety evaluation and multi-parameter arrhythmia profiling of cardiomyocytes using microelectrode arrays. Toxicol Appl Pharmacol 288, 249-257.

Gintant, G., 2011. An evaluation of hERG current assay performance: Translating preclinical safety studies to clinical QT prolongation. Pharmacol Ther 129, 109-119.

Gonze, D., Halloy, J., Goldbeter, A., 2002. Robustness of circadian rhythms with respect to molecular noise. Proc Natl Acad Sci U S A 99, 673-678.

Gualdani, R., Tadini-Buoninsegni, F., Roselli, M., Defrenza, I., Contino, M., Colabufo, N.A., Lentini, G., 2015. Inhibition of hERG potassium channel by the antiarrhythmic agent mexiletine and its metabolite m-hydroxymexiletine. Pharmacol Res Perspect 3, e00160.

Guo, J., Zhan, S., Lees-Miller, J.P., Teng, G., Duff, H.J., 2005. Exaggerated block of hERG (KCNH2) and prolongation of action potential duration by erythromycin at temperatures between 37 degrees C and 42 degrees C. Heart Rhythm 2, 860-866.

Guo, L., Abrams, R.M., Babiarz, J.E., Cohen, J.D., Kameoka, S., Sanders, M.J., Chiao, E., Kolaja, K.L., 2011. Estimating the risk of drug-induced proarrhythmia using human induced pluripotent stem cellderived cardiomyocytes. Toxicol Sci 123, 281-289.

Guo, L., Coyle, L., Abrams, R.M., Kemper, R., Chiao, E.T., Kolaja, K.L., 2013. Refining the human iPSCcardiomyocyte arrhythmic risk assessment model. Toxicol Sci 136, 581-594.

Harris, K., Aylott, M., Cui, Y., Louttit, J.B., McMahon, N.C., Sridhar, A., 2013. Comparison of electrophysiological data from human-induced pluripotent stem cell-derived cardiomyocytes to functional preclinical safety assays. Toxicol Sci 134, 412-426.

Hartman, M.E., Dai, D.F., Laflamme, M.A., 2015. Human pluripotent stem cells: Prospects and challenges as a source of cardiomyocytes for in vitro modeling and cell-based cardiac repair. Adv Drug Deliv Rev. 
Huang, X.P., Mangano, T., Hufeisen, S., Setola, V., Roth, B.L., 2010. Identification of human Ether-a-go-go related gene modulators by three screening platforms in an academic drug-discovery setting. Assay Drug Dev Technol 8, 727-742.

Jonsson, M.K., Wang, Q.D., Becker, B., 2011. Impedance-based detection of beating rhythm and proarrhythmic effects of compounds on stem cell-derived cardiomyocytes. Assay Drug Dev Technol 9, 589-599.

Kamen, P.W., Krum, H., Tonkin, A.M., 1996. Poincare plot of heart rate variability allows quantitative display of parasympathetic nervous activity in humans. Clin Sci (Lond) 91, 201-208.

Kantelhardt, J.W., Koscielny-Bunde, E., Rego, H.H.A., Havlin, S., Bunde, A., 2001. Detecting long-range correlations with detrended fluctuation analysis. Physica A 295, 441-454.

Karmakar, C.K., Khandoker, A.H., Gubbi, J., Palaniswami, M., 2009. Complex correlation measure: a novel descriptor for Poincare plot. Biomed Eng Online 8, 17.

Kirsch, G.E., Trepakova, E.S., Brimecombe, J.C., Sidach, S.S., Erickson, H.D., Kochan, M.C., Shyjka, L.M., Lacerda, A.E., Brown, A.M., 2004. Variability in the measurement of hERG potassium channel inhibition: effects of temperature and stimulus pattern. J Pharmacol Toxicol Methods 50, 93101.

Kolaja, K., 2014. Stem cells and stem cell-derived tissues and their use in safety assessment. J Biol Chem 289, 4555-4561.

Kramer, J., Obejero-Paz, C.A., Myatt, G., Kuryshev, Y.A., Bruening-Wright, A., Verducci, J.S., Brown, A.M., 2013. MICE models: superior to the HERG model in predicting Torsade de Pointes. Sci Rep 3, 2100.

Lacerda, A.E., Kuryshev, Y.A., Chen, Y., Renganathan, M., Eng, H., Danthi, S.J., Kramer, J.W., Yang, T., Brown, A.M., 2008. Alfuzosin delays cardiac repolarization by a novel mechanism. J Pharmacol Exp Ther 324, 427-433.

Lee, B.H., Lee, S.H., Chu, D., Hyun, J.W., Choe, H., Choi, B.H., Jo, S.H., 2011. Effects of the histamine H(1) receptor antagonist hydroxyzine on $\mathrm{hERG} \mathrm{K}(+)$ channels and cardiac action potential duration. Acta Pharmacol Sin 32, 1128-1137.

Lu, H.R., Vlaminckx, E., Hermans, A.N., Rohrbacher, J., Van Ammel, K., Towart, R., Pugsley, M., Gallacher, D.J., 2008. Predicting drug-induced changes in QT interval and arrhythmias: QT-shortening drugs point to gaps in the ICHS7B Guidelines. Br J Pharmacol 154, 1427-1438.

Luo, T., Luo, A., Liu, M., Liu, X., 2008. Inhibition of the HERG channel by droperidol depends on channel gating and involves the $S 6$ residue F656. Anesth Analg 106, 1161-1170, table of contents.

Mathur, A., Loskill, P., Shao, K., Huebsch, N., Hong, S., Marcus, S.G., Marks, N., Mandegar, M., Conklin, B.R., Lee, L.P., Healy, K.E., 2015. Human iPSC-based cardiac microphysiological system for drug screening applications. Sci Rep 5, 8883.

Nozaki, Y., Honda, Y., Tsujimoto, S., Watanabe, H., Kunimatsu, T., Funabashi, H., 2014. Availability of human induced pluripotent stem cell-derived cardiomyocytes in assessment of drug potential for QT prolongation. Toxicol Appl Pharmacol 278, 72-77.

Nozaki, Y., Honda, Y., Watanabe, H., Saiki, S., Koyabu, K., Itoh, T., Nagasawa, C., Nakamori, C., Nakayama, C., Iwasaki, H., Suzuki, S., Washio, I., Takahashi, E., Miyamoto, K., Yamanishi, A., Endo, H., Shinozaki, J., Nogawa, H., Kunimatsu, T., 2016. CSAHi study: Validation of multi-electrode array systems (MEA60/2100) for prediction of drug-induced proarrhythmia using human iPS cellderived cardiomyocytes -assessment of inter-facility and cells lot-to-lot-variability. Regul Toxicol Pharmacol 77, 75-86.

Paul, A.A., Witchel, H.J., Hancox, J.C., 2002. Inhibition of the current of heterologously expressed HERG potassium channels by flecainide and comparison with quinidine, propafenone and lignocaine. Br J Pharmacol 136, 717-729. 
Peng, S., Lacerda, A.E., Kirsch, G.E., Brown, A.M., Bruening-Wright, A., 2010. The action potential and comparative pharmacology of stem cell-derived human cardiomyocytes. J Pharmacol Toxicol Methods 61, 277-286.

Pikovsky, A., Zaks, M., Rosenblum, M., Osipov, G., Kurths, J., 1997. Phase synchronization of chaotic oscillations in terms of periodic orbits. Chaos 7, 680-687.

Pradhapan, P., Kuusela, J., Viik, J., Aalto-Setala, K., Hyttinen, J., 2013. Cardiomyocyte MEA data analysis (CardioMDA)--a novel field potential data analysis software for pluripotent stem cell derived cardiomyocytes. PLoS One 8, e73637.

Qi, F., Hou, Z., Xin, H., 2003a. New characterization of disorder taming spatiotemporal chaos. Phys Lett A 308, 405-410.

Qi, F., Hou, Z., Xin, H., 2003b. Ordering chaos by random shortcuts. Phys Rev Lett 91, 064102.

Redfern, W.S., Carlsson, L., Davis, A.S., Lynch, W.G., MacKenzie, I., Palethorpe, S., Siegl, P.K., Strang, I., Sullivan, A.T., Wallis, R., Camm, A.J., Hammond, T.G., 2003. Relationships between preclinical cardiac electrophysiology, clinical QT interval prolongation and torsade de pointes for a broad range of drugs: evidence for a provisional safety margin in drug development. Cardiovasc Res $58,32-45$.

Sager, P.T., Gintant, G., Turner, J.R., Pettit, S., Stockbridge, N., 2014. Rechanneling the cardiac proarrhythmia safety paradigm: a meeting report from the Cardiac Safety Research Consortium. Am Heart J 167, 292-300.

Scholz, E.P., Konrad, F.M., Weiss, D.L., Zitron, E., Kiesecker, C., Bloehs, R., Kulzer, M., Thomas, D., Kathofer, S., Bauer, A., Maurer, M.H., Seemann, G., Katus, H.A., Karle, C.A., 2007. Anticholinergic antiparkinson drug orphenadrine inhibits HERG channels: block attenuation by mutations of the pore residues Y652 or F656. Naunyn Schmiedebergs Arch Pharmacol 376, 275-284.

Scott, C.W., Zhang, X., Abi-Gerges, N., Lamore, S.D., Abassi, Y.A., Peters, M.F., 2014. An impedancebased cellular assay using human iPSC-derived cardiomyocytes to quantify modulators of cardiac contractility. Toxicol Sci 142, 331-338.

Sirenko, O., Cromwell, E.F., Crittenden, C., Wignall, J.A., Wright, F.A., Rusyn, I., 2013. Assessment of beating parameters in human induced pluripotent stem cells enables quantitative in vitro screening for cardiotoxicity. Toxicol Appl Pharmacol 273, 500-507.

Synnergren, J., Ameen, C., Jansson, A., Sartipy, P., 2012. Global transcriptional profiling reveals similarities and differences between human stem cell-derived cardiomyocyte clusters and heart tissue. Physiol Genomics 44, 245-258.

Takens, F., 1981. Detecting strange attractors in turbulence. Springer New York, NY.

Tarantino, P., Appleton, N., Lansdell, K., 2005. Effect of trazodone on hERG channel current and QTinterval. Eur J Pharmacol 510, 75-85.

Teschemacher, A.G., Seward, E.P., Hancox, J.C., Witchel, H.J., 1999. Inhibition of the current of heterologously expressed HERG potassium channels by imipramine and amitriptyline. $\mathrm{Br} \mathrm{J}$ Pharmacol 128, 479-485.

Tie, H., Walker, B.D., Singleton, C.B., Valenzuela, S.M., Bursill, J.A., Wyse, K.R., Breit, S.N., Campbell, T.J., 2000. Inhibition of HERG potassium channels by the antimalarial agent halofantrine. $\mathrm{Br} J$ Pharmacol 130, 1967-1975.

Vigneault, P., Kaddar, N., Bourgault, S., Caillier, B., Pilote, S., Patoine, D., Simard, C., Drolet, B., 2011. Prolongation of cardiac ventricular repolarization under paliperidone: how and how much? J Cardiovasc Pharmacol 57, 690-695.

Wang, T., Hu, N., Cao, J., Wu, J., Su, K., Wang, P., 2013. A cardiomyocyte-based biosensor for antiarrhythmic drug evaluation by simultaneously monitoring cell growth and beating. Biosens Bioelectron 49, 9-13. 
Xi, B., Wang, T., Li, N., Ouyang, W., Zhang, W., Wu, J., Xu, X., Wang, X., Abassi, Y.A., 2011. Functional cardiotoxicity profiling and screening using the xCELLigence RTCA Cardio System. J Lab Autom 16, 415-421.

Yap, Y.G., Camm, A.J., 2003. Drug induced QT prolongation and torsades de pointes. Heart 89, 13631372.

Yun, J., Chung, E., Choi, K.H., Cho, D.H., Song, Y.J., Han, K.M., Cha, H.J., Shin, J.S., Seong, W.K., Kim, Y.H., Kim, H.S., 2015. Cardiovascular Safety Pharmacology of Sibutramine. Biomol Ther (Seoul) 23, 386-389.

Zhang, Y.H., Cheng, H., Alexeenko, V.A., Dempsey, C.E., Hancox, J.C., 2010. Characterization of recombinant $h E R G \mathrm{~K}(+)$ channel inhibition by the active metabolite of amiodarone desethylamiodarone. J Electrocardiol 43, 440-448.

Zhou, Z., Gong, Q., Ye, B., Fan, Z., Makielski, J.C., Robertson, G.A., January, C.T., 1998. Properties of HERG channels stably expressed in HEK 293 cells studied at physiological temperature. Biophys J 74, 230-241. 
Kirby et al_Figure 1

2-column

Color on line only

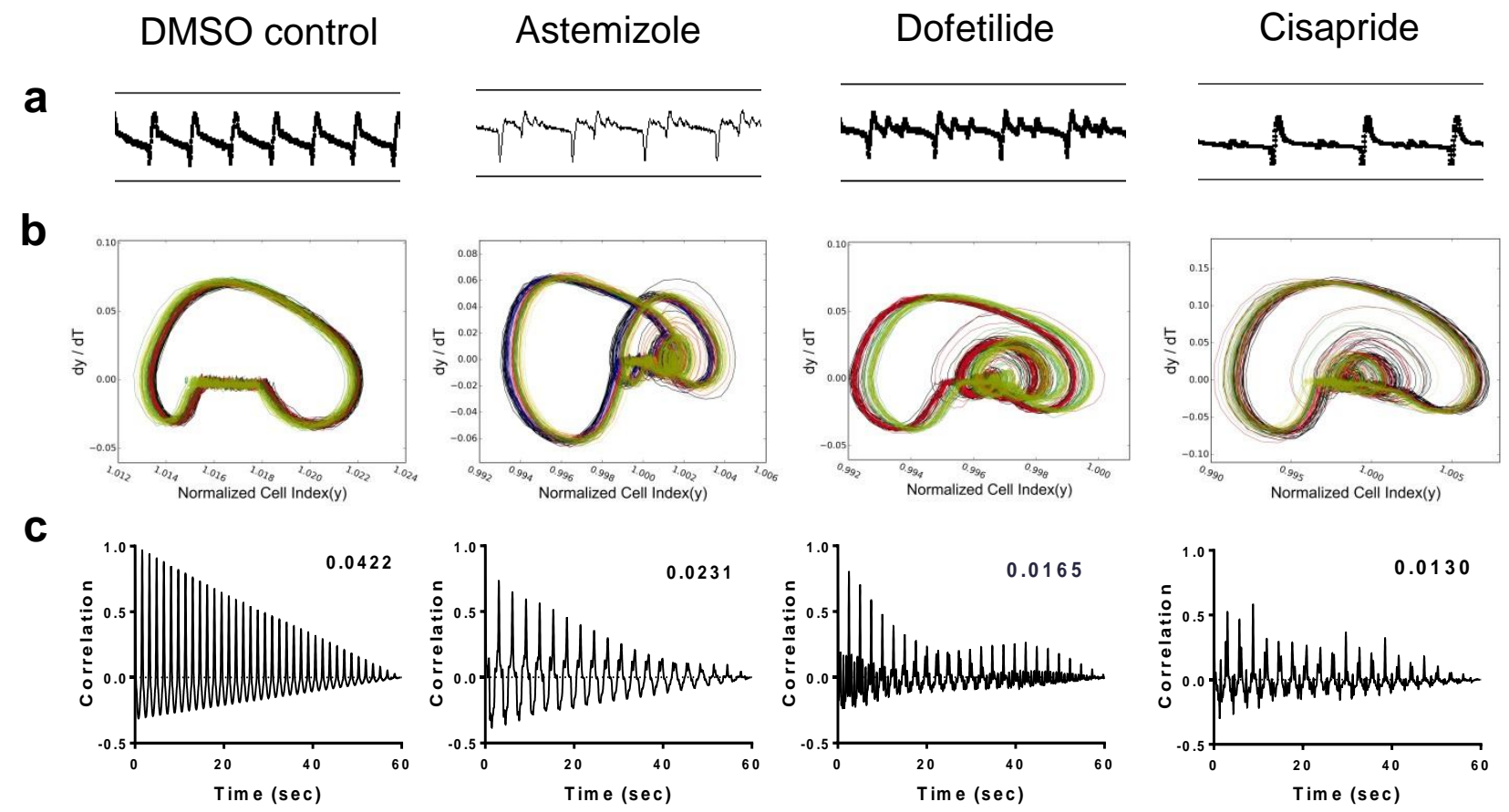


Kirby et al_Figure 2

2-column
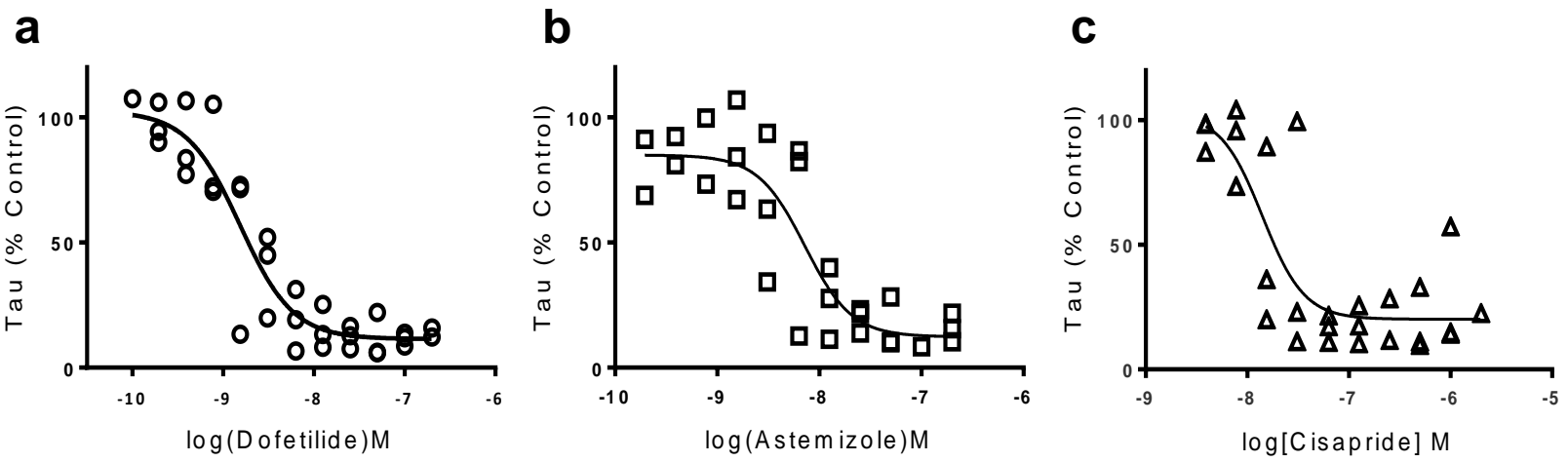
Kirby et al_Figure 3

2-column

print in color

a

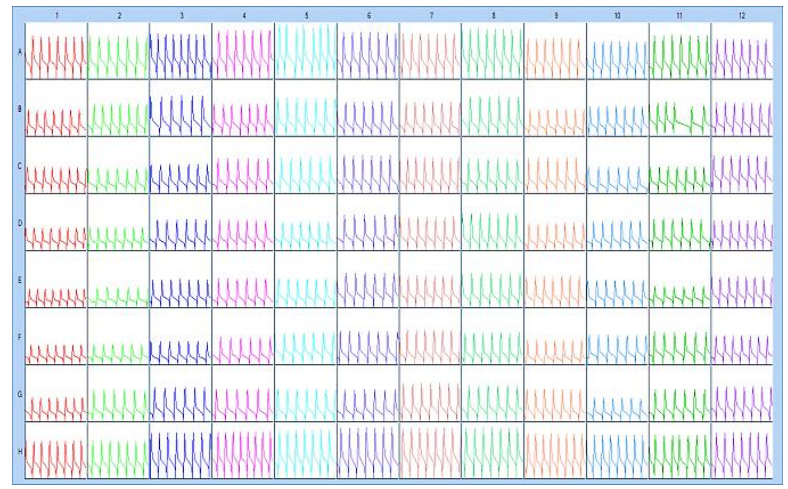

C

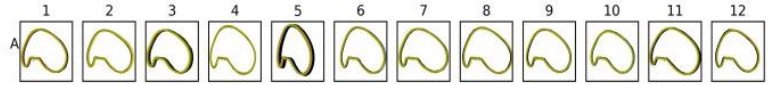

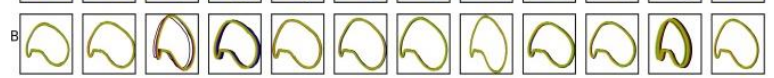
¿DDQDDDDODOD

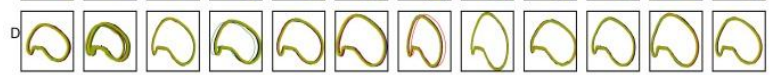

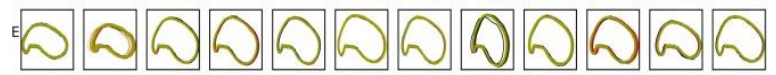

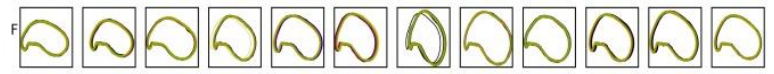

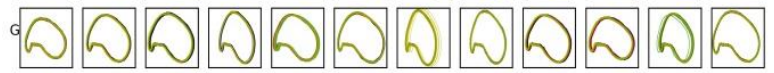

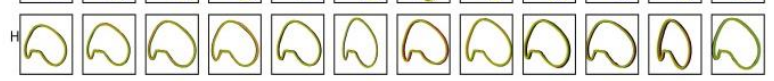

e

$\begin{array}{llllllllllll}1 & 2 & 3 & 4 & 5 & 6 & 7 & 8 & 9 & 10 & 11 & 12\end{array}$

\begin{tabular}{llllllllllll|l|l|l|l|} 
A & 0.053 & 0.040 & 0.051 & 0.053 & 0.030 & 0.050 & 0.057 & 0.054 & 0.052 & 0.051 & 0.053 & 0.052 \\
\hline
\end{tabular}

\begin{tabular}{llllllllllllllll} 
B & 0.053 & 0.054 & 0.035 & 0.025 & 0.044 & 0.052 & 0.050 & 0.049 & 0.043 & 0.050 & 0.010 & 0.052 \\
\hline
\end{tabular}

$\begin{array}{lllllllllllllllll}C & 0.050 & 0.043 & 0.048 & 0.049 & 0.049 & 0.049 & 0.050 & 0.047 & 0.049 & 0.042 & 0.045 & 0.046\end{array}$

$\begin{array}{llllllllllllllll}\text { D } & 0.051 & 0.020 & 0.049 & 0.027 & 0.045 & 0.043 & 0.047 & 0.051 & 0.049 & 0.050 & 0.045 & 0.050\end{array}$

\begin{tabular}{lllllllllllllllll}
$\mathrm{E}$ & 0.051 & 0.021 & 0.050 & 0.046 & 0.050 & 0.047 & 0.050 & 0.031 & 0.030 & 0.036 & 0.040 & 0.053 \\
\hline
\end{tabular}

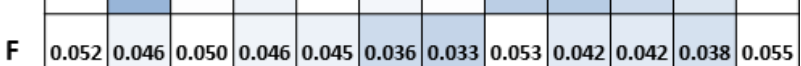

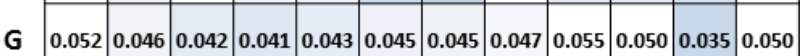

\begin{tabular}{llllllllllll|l|l|l|} 
H & 0.056 & 0.050 & 0.052 & 0.051 & 0.056 & 0.058 & 0.056 & 0.042 & 0.055 & 0.054 & 0.034 & 0.051 \\
\hline
\end{tabular} b

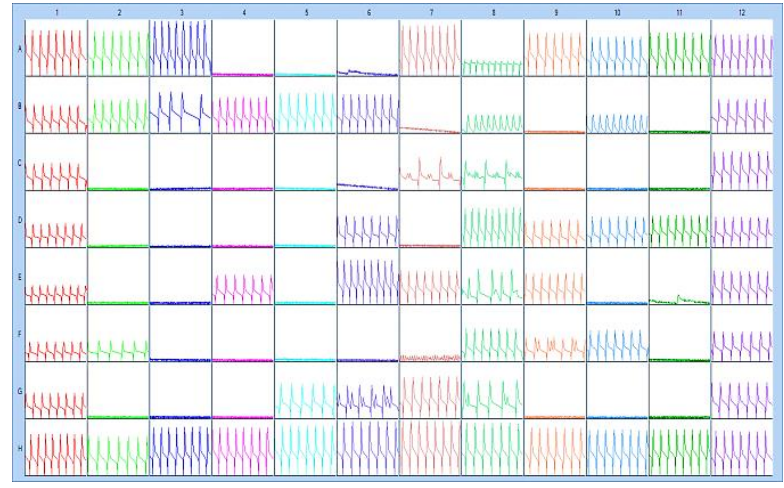

d

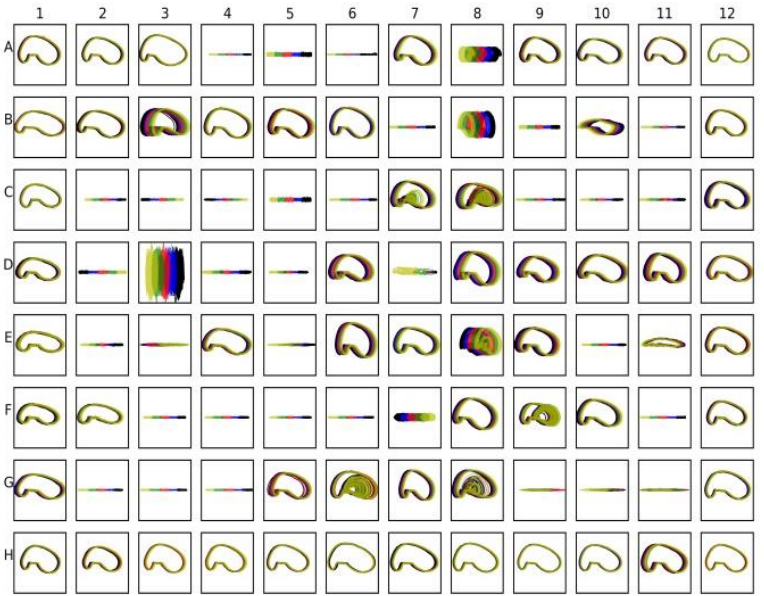

f

$\begin{array}{llllllllllll}1 & 2 & 3 & 4 & 5 & 6 & 7 & 8 & 9 & 10 & 11 & 12\end{array}$

\begin{tabular}{l|l|l|l|l|l|l|l|l|l|l|l|l|}
$A$ & 0.044 & 0.044 & 0.072 & ND & ND & ND & 0.058 & 0.026 & 0.046 & 0.049 & 0.047 & 0.044
\end{tabular}

\begin{tabular}{l|l|l|l|l|l|l|l|l|l|l|l|l} 
B & 0.048 & 0.049 & 0.015 & 0.049 & 0.042 & 0.051 & ND & 0.025 & ND & ND & ND & 0.038
\end{tabular}

\begin{tabular}{l|l|l|l|l|l|l|l|l|l|l|l|l} 
C & 0.040 & ND & ND & ND & ND & ND & 0.017 & 0.005 & ND & ND & ND & 0.041
\end{tabular}

\begin{tabular}{l|l|l|l|l|l|l|l|l|l|l|l|l}
$D$ & 0.044 & ND & 0.065 & ND & ND & 0.042 & ND & 0.052 & 0.047 & 0.046 & 0.043 & 0.044
\end{tabular}

\begin{tabular}{|l|l|l|l|l|l|l|l|l|l|l|l|l}
\hline E & 0.040 & ND & ND & 0.045 & 0.030 & 0.060 & 0.045 & 0.012 & 0.036 & ND & 0.039 & 0.047
\end{tabular}

\begin{tabular}{lll|l|l|l|l|l|l|l|l|l|l|l}
\hline & 0.041 & 0.033 & ND & ND & ND & ND & ND & 0.051 & 0.012 & 0.047 & ND & 0.040
\end{tabular}

\begin{tabular}{l|l|l|l|l|l|l|l|l|l|l|l|l} 
G & 0.044 & ND & ND & ND & 0.026 & 0.011 & 0.048 & 0.015 & ND & ND & ND & 0.045 \\
\hline
\end{tabular}

$\begin{array}{llllllllllllllllll}\text { H } & 0.050 & 0.039 & 0.045 & 0.035 & 0.048 & 0.051 & 0.052 & 0.045 & 0.051 & 0.046 & 0.043 & 0.045\end{array}$ 
Kirby et al_Figure 4 1-column
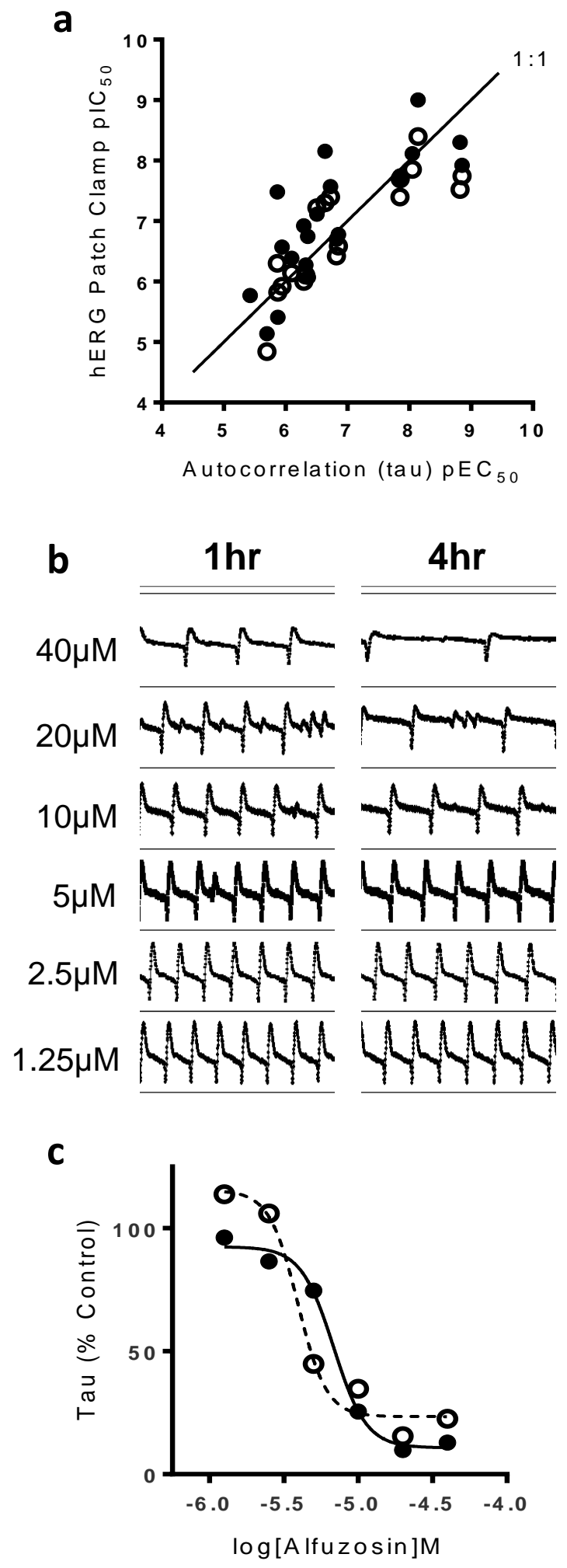
Kirby et al_Figure 5

1-column

Color on line only

a

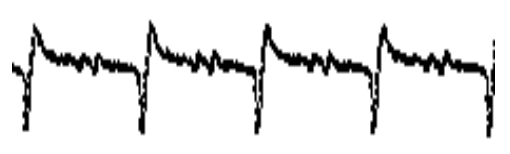

b

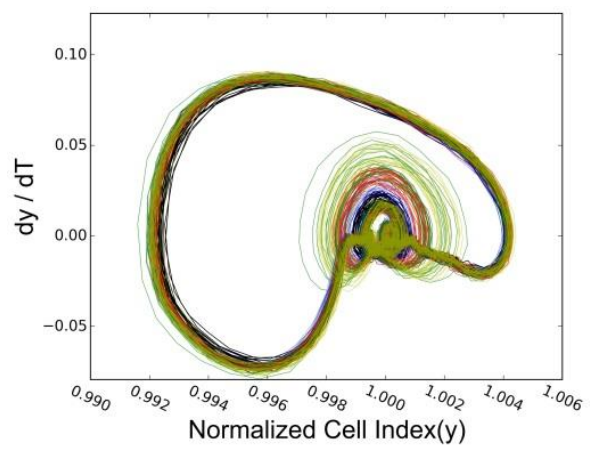

C

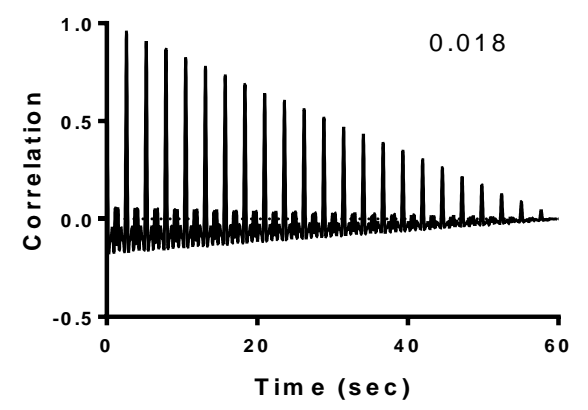

d

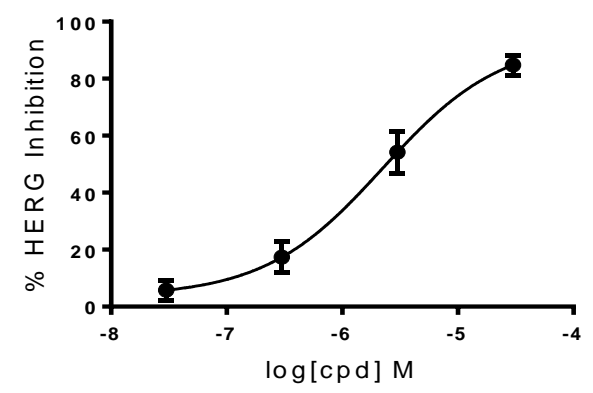



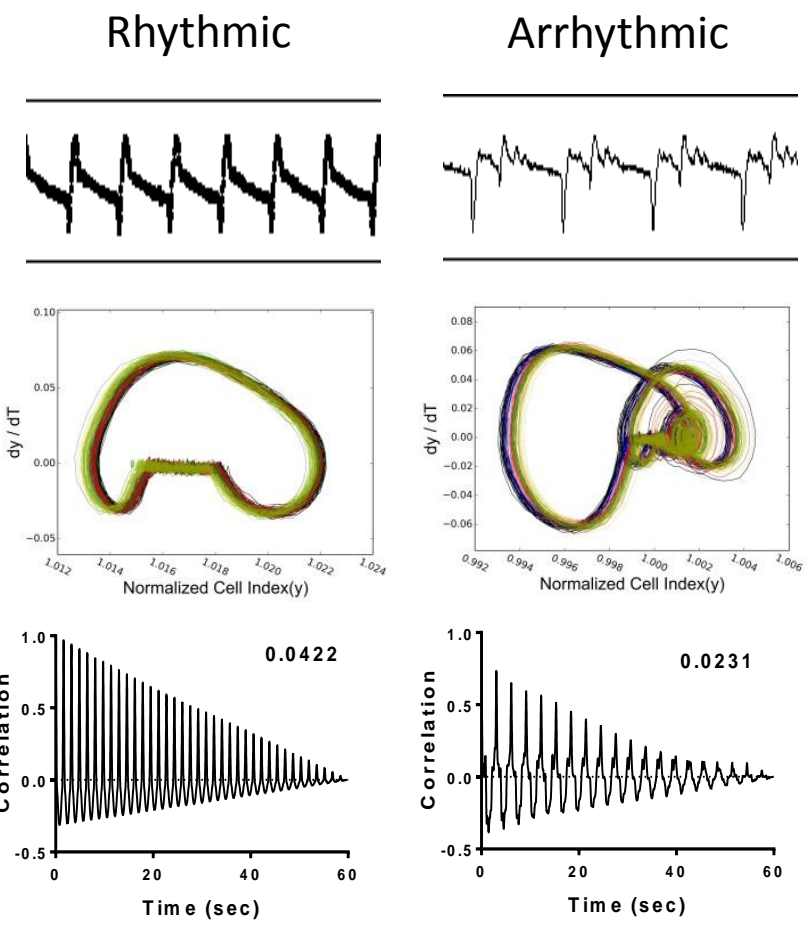